\title{
Psychosocial impact of the COVID-19 pandemic on paediatric healthcare workers
}

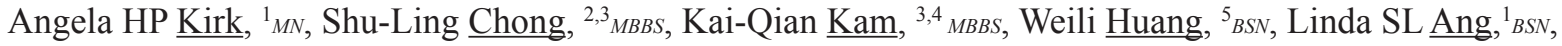
Jan Hau Lee, ${ }^{3,6}{ }_{M B B S}$, Rehena Sultana, ${ }^{7}$ MSC (Statss), Kam Lun $\underline{\text { Hon, }},{ }^{8}$ MBBS, Judith JM Wong, ${ }^{3,6}{ }_{M B B C h} B A O$

\begin{abstract}
Introduction: Frontline healthcare workers (HCWs) exposed to coronavirus disease 2019 (COVID-19) are at risk of psychological distress. This study evaluates the psychological impact of COVID-19 pandemic on HCWs in a national paediatric referral centre.

Methods: This was a survey-based study that collected demographic, work environment and mental health data from paediatric HCWs in the emergency, intensive care and infectious disease units. Psychological impact was measured using the Depression, Anxiety, Stress Scale-21. Multivariate regression analysis was performed to identify risk factors associated with psychological distress.

Results: The survey achieved a response rate of $93.9 \%$ (430 of 458). Of the 430 respondents, symptoms of depression, anxiety and stress were reported in $168(39.1 \%), 205(47.7 \%)$ and $106(24.7 \%)$, respectively. Depression was reported in the mild (47, 10.9\%), moderate $(76,17.7 \%)$, severe $(23,5.3 \%)$ and extremely severe $(22,5.1 \%)$ categories. Anxiety $(205,47.7 \%)$ and stress $(106,24.7 \%)$ were reported in the mild category only. Collectively, regression analysis identified female sex, a perceived lack of choice in work scope/environment, lack of protection from COVID-19, lack of access to physical activities and rest, the need to perform additional tasks, and the experience of stigma from the community as risk factors for poor psychological outcome.

Conclusion: A high prevalence of depression, anxiety and stress was reported among frontline paediatric HCWs during the COVID-19 pandemic. Personal psychoneuroimmunity and organisational prevention measures can be implemented to lessen psychiatric symptoms. At the national level, involving mental health professionals to plan and coordinate psychological intervention for the country should be considered.
\end{abstract}

Ann Acad Med Singap 2021;50:203-11

Keywords: Anxiety, depression, healthcare worker, medical staff, psychological, stress

\section{INTRODUCTION}

Coronavirus disease 2019 (COVID-19) was first reported in December 2019 and has since evolved into a global pandemic, infecting millions of people and causing more than 2.7 million deaths. ${ }^{1,2}$ Early studies done in China during this COVID-19 pandemic have shown considerable mental health impact on healthcare workers (HCWs), especially those working on the frontline. ${ }^{3,4}$ HCWs exposed directly to COVID-19 may be affected not only by fears of contracting the virus and spreading it to their loved ones, but also by work-related factors including the lack of manpower, increased working hours, inadequate personal protective equipment, difficult triage decisions and difficult isolation environments. ${ }^{3,5,6}$ An international collaborative survey conducted in Asia Pacific, which was independent of the COVID-19 disease burden within the country, demonstrated varying levels of mental health burden among HCWs. ${ }^{7}$ Moreover,

\footnotetext{
${ }^{1}$ Children's Intensive Care Unit, Division of Nursing, KK Women's and Children's Hospital, Singapore

${ }^{2}$ Department of Emergency Medicine, Division of Medicine, KK Women's and Children's Hospital, Singapore

${ }^{3}$ Duke-NUS Medical School, Singapore

${ }^{4}$ Infectious Disease Service, Division of Medicine, KK Women's and Children's Hospital, Singapore

${ }^{5}$ Department of Emergency Medicine, Division of Nursing, KK Women's and Children's Hospital, Singapore

${ }^{6}$ Children's Intensive Care Unit, Division of Medicine, KK Women's and Children's Hospital, Singapore

${ }^{7}$ Centre for Quantitative Medicine, SingHealth Duke-NUS, Singapore

${ }^{8}$ Department of Paediatrics, The Chinese University of Hong Kong, Hong Kong

Correspondence: Angela HP Kirk, Children's Intensive Care unit, KK Women's and Children's Hospital, 100 Bukit Timah Road, Singapore 229899.

Email: angela.kirk.hui.p@kkh.com.sg
} 


\section{CLINICAL IMPACT}

\section{What is New}

- This survey showed a high prevalence of symptoms of depression, anxiety and stress among frontline paediatric healthcare workers during the COVID-19 pandemic.

- Modifiable risk factors included a perceived lack of choice of work, lack of protection from COVID-19, lack of access to physical activities and rest, the need to perform additional tasks, and the experience of stigma from the community.

\section{Clinical Implications}

- This study indicates that the psychological impact on high-risk healthcare workers in paediatric hospitals was substantial, despite the relatively mild clinical impact of COVID-19 on the paediatric population.

- Data from this study may guide future efforts to improve personal psychoneuroimmunity, organisational prevention and interventional measures to lessen psychiatric symptoms during crises.

physical symptoms including headache, lethargy and insomnia have also been reported in a considerable proportion of HCWs and were associated with their psychological states. ${ }^{8}$

Reports on the psychological impact on HCWs from paediatric hospitals, however, are relatively scarce. This lack of study may be due to the relatively lower prevalence and generally milder disease severity of paediatric COVID-19 compared to adults, ${ }^{9}$ resulting in greater focus being diverted to adult hospitals. Nevertheless, the psychological impact may be independent of disease burden. ${ }^{7}$ An early multicentre study among paediatric medical staff in China highlighted that paediatric HCWs are also vulnerable to adverse psychological impact. ${ }^{10}$

Singapore reported its first imported case of COVID-19 on 23 January 2020, and our first paediatric COVID-19 case was reported on 4 February 2020. ${ }^{11}$ To date, approximately 60,000 COVID-19 cases have been confirmed in Singapore. ${ }^{12}$ Of note, all paediatric COVID-19 patients from our hospital survived, none required ICU admission and all were discharged without complication. Although the clinical impact of
COVID-19 on the paediatric population is mild, we hypothesised that the psychological impact on highrisk HCWs in paediatric hospitals would be substantial. In this study, we aimed to evaluate the psychological impact of the COVID-19 pandemic on HCWs in a national paediatric referral centre.

\section{METHODS}

This study was an electronic survey administered by email invitation to eligible participants. All data collected were completed in an anonymised and voluntary fashion. Reminders were sent via mass e-mail and token reward vouchers were given as an incentive to increase the response rate. Reporting was in accordance with the Checklist for Reporting Results of Internet E-Surveys guidelines. ${ }^{13}$ This study received exemption from the ethics board review.

\section{Participating sites and target population}

This study was conducted at KK Women's and Children's Hospital, which is a standalone children's hospital and the main referral centre for paediatric COVID-19 cases in Singapore. The target population was high-risk HCWs comprising medical and nursing professionals who were part of the paediatric intensive care unit (ICU), children's emergency (CE) and infectious disease (ID) teams in direct contact with confirmed or suspected COVID-19 cases, as these cases were considered at greatest risk of developing adverse psychological outcomes. ${ }^{14}$ Since January 2020, the hospital has made changes to the workflows and protocols in preparation for the COVID-19 outbreak. The isolation facilities were boosted in numbers and equipped with critical care paraphernalia, in anticipation of admitting COVID-19 patients requiring intermediate care or ICU management. ${ }^{15,16}$ ICU staff deployment had to be doubled to cope with routine care as well as anticipate a surge in COVID-19 admissions. ${ }^{15}$ Concurrent changes in our CE department included the building of a temporary, pre-triage screening facility and alterations of staffing schedules into modular teams in 12-hour shifts to prevent cross-exposure and hospital transmission. ${ }^{17}$ ID physicians led the hospital on COVID-19 preparedness, planned workflows, responded to clinical queries relating to COVID-19, and managed the care of suspected or confirmed COVID-19 cases. ICU, CE and ID HCWs were surveyed from 28 April 2020 to 5 May 2020. The survey period was midway ( 3 weeks) through a nationwide lockdown period (6 weeks)..$^{18,19}$ 


\section{Self-analysis questionnaire}

The Depression, Anxiety, Stress Scale (DASS) is a selfreported inventory to measure the negative emotional states of depression, anxiety and stress. ${ }^{20}$ The original 42-item scale was abbreviated to a 21 -item short version (DASS-21), ${ }^{21}$ which received further validation in clinical cohorts, including Asian cohorts during the COVID-19 pandemic. ${ }^{10,22,23}$ In addition to DASS-21, subjects were surveyed on sociodemographic factors, work environment and daily lifestyle. Both closedended and open-ended questions were used to collect information on work environment and lifestyle factors. All closed-ended questions were mandatory, whereas optional open-ended questions were used to solicit additional opinions or comments. Instructions were included in the survey and participants were reminded to submit only 1 response.

\section{Statistical analyses}

Sociodemographic characteristics and work environment factors were described for the medical and nursing cohorts in the ICU, CE and ID teams. Categorical variables are presented as counts and percentages, and continuous variables as median and interquartile range. The median DASS-21 scores and prevalence of depression, anxiety and psychological stress of the medical cohort were compared with those of the nursing cohort of the frontline HCWs. These comparisons were repeated among the ICU, CE and ID teams. The main outcomes were the prevalence of depression, anxiety and stress among frontline HCWs and were treated as binary data. Depression, anxiety or stress was considered present if scores exceeded the normal cut-off, and was categorised as mild, moderate, severe and extremely severe based on published cut-offs. ${ }^{24}$ The Kruskal-Wallis and chi-square tests were used to compare continuous and categorical variables, respectively.

Univariate and multivariable logistic regression models were used to quantify the association of demographic and work-environment risk factors with the primary outcome. The association was described using odds ratio (OR) with $95 \%$ confidence intervals (CI). Variables with $P$ value $<0.2$ in the univariate model were selected for the multivariable model. Union of the variables selected in the forward, backward and stepwise methods were then used to finalise the variables list in the multivariable model with entry criteria and stay criteria of 0.2 and 0.05 , respectively. Separate univariate and multivariable models were also fitted for secondary outcomes, that is, anxiety and stress. All tests were 2 -tailed and $P$ value $<0.05$ was accepted as statistically significant. Analyses were performed using Stata software version 15.1 (StataCorp, College Station, US) and SAS software version 9.3 (SAS Institute, Cary, US).

\section{RESULTS}

This survey achieved a response rate of 93.9\% (430 of 458). Of the 430 respondents, 175 (40.7\%) were medical staff and the remainder were nursing staff. The sociodemographic profile and work environment characteristics of the HCWs are described in Tables 1 and 2 , respectively.

The majority of participants had come into contact with suspected or confirmed cases of COVID-19 at the time of this study. Sixty-nine of 430 respondents $(16.0 \%)$ were required to perform tasks in addition to their usual work routine (e.g. infection control, administrative and training tasks in various settings, and housekeeping tasks in the isolation wards). However, only 35 (8.1\%) felt uncomfortable with these additional tasks. Eighty-seven respondents (20.2\%) perceived a lack of choice with regard to the tasks they were required to perform, and the highest incidence of this perception were from the ICU nursing cohort (23 of 67 [34.3\%]); they reported a perceived lack of choice in nursing suspected or confirmed critical cases of COVID-19. Majority of frontline HCWs felt protected from contracting the virus and had access to basic needs like rest, healthy food or beverages, and exercise. However, of the 430 respondents, 129 (30.0\%) perceived a lack of time and access to stay in contact with friends and family, and 100 (23.3\%) perceived avoidance by family members or their community owing to stigma and fear of contracting COVID-19 from them (e.g. avoidance from family members, friends, neighbours and taxi drivers). Participants expressed that the closure of sports facilities, lack of time and insufficient rest had contributed to the lack of access to and time for physical activities. With the nationwide lockdown, the mandate for social distancing and travel prohibition for returning to overseas family members had prevented them from staying in contact with family and friends during this pandemic.

Among the 430 respondents, the prevalence of mild, moderate, severe and extremely severe depression based on the DASS-21 categories were $10.9 \%(n=47), 17.7 \%$ $(n=76), 5.3 \%(n=23)$ and $5.1 \%(n=22)$, respectively. There were no differences in the median depression factor scores (interquartile range) between the medical 

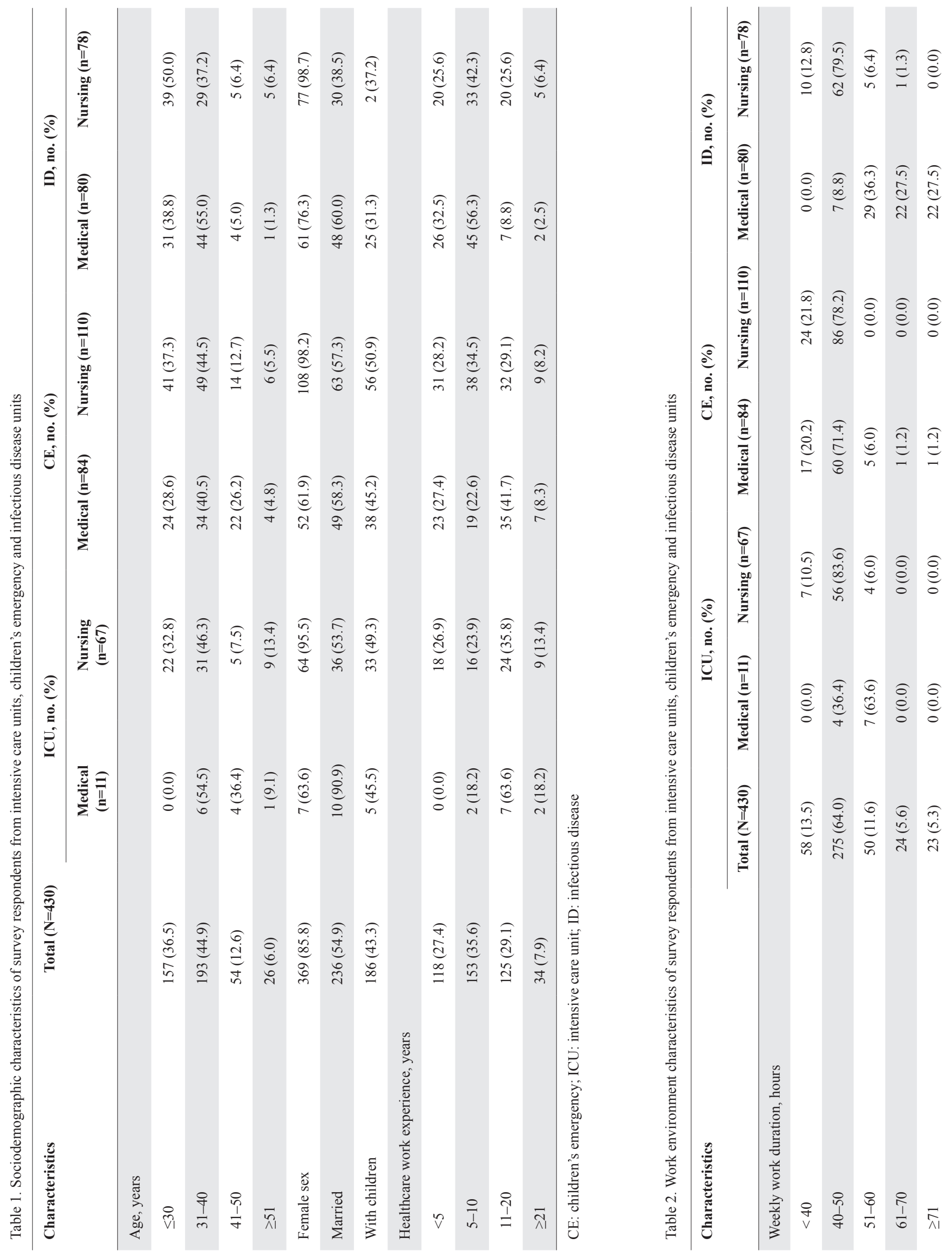

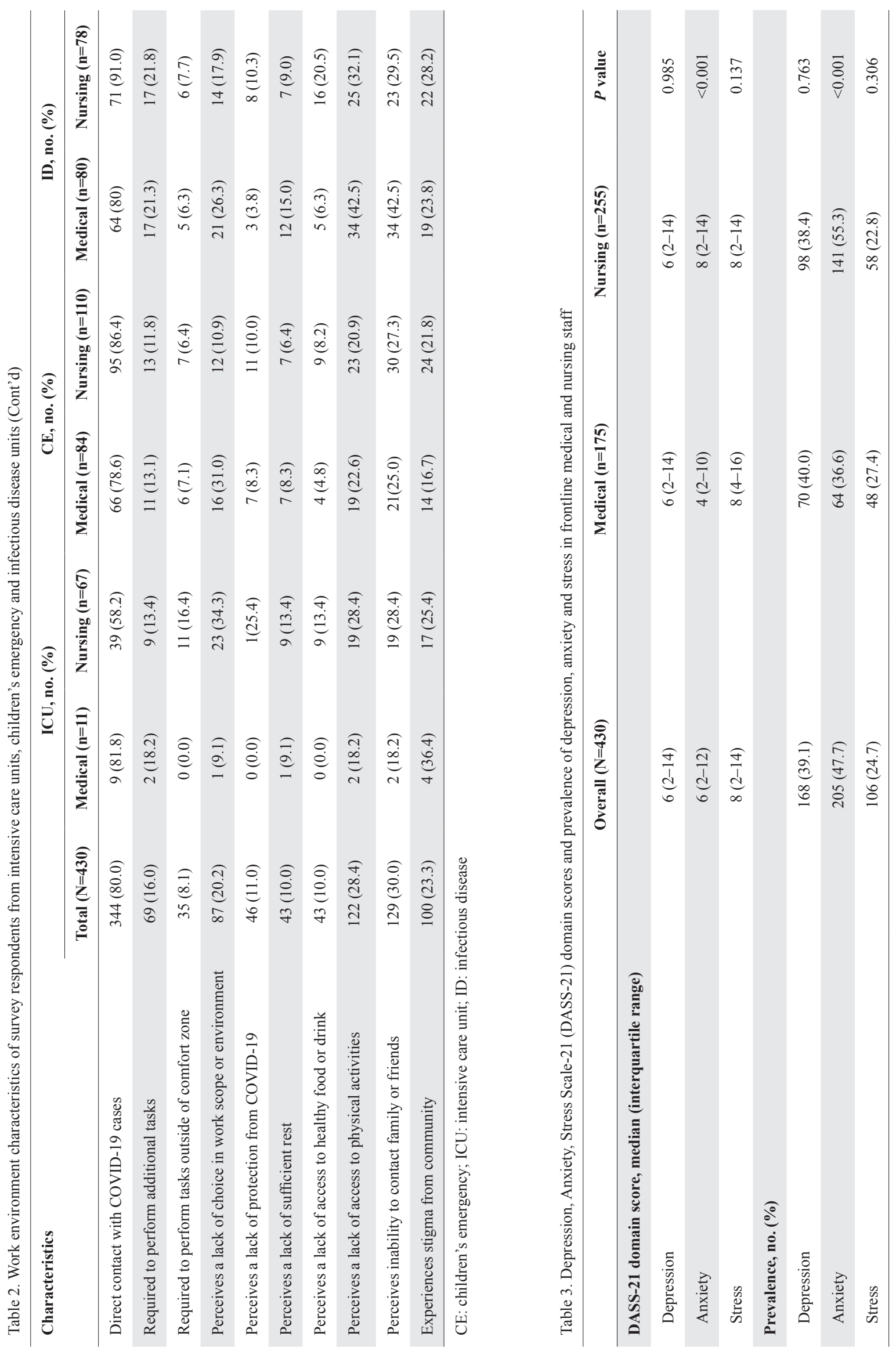
and nursing cohorts (Table 3 ) or among the ICU, CE and ID teams (5 [2-12], 6 [2-14] and 6 [2-14], respectively; $P=0.547)$. Anxiety was found in the mild category only, and its prevalence was $47.7 \%$ (205 of 430). The median anxiety factor score was higher in the nursing cohort than that in the medical cohort (Table 3), but the median score (interquartile range) was not significantly different among the ICU, CE and ID teams (8 [2-12], 6 [2-14] and 6 [2-10], respectively; $P=0.320$ ). Lastly, psychological stress was also only found in the mild category, and its prevalence was $24.7 \%$ (106 of 430$)$. There were no differences in the median stress factor scores (interquartile range) between the medical and nursing cohorts (Table 3 ) or among the ICU, CE and ID teams (8 [2-16], 8 [2-16] and 8 [2-14], respectively; $P=0.975$ ). Respondents cited clear communication from hospital leaders; availability of adequate personal protective equipment; and support from the hospital administration, colleagues, family and religious observances as helping to relieve stress during this pandemic. Uncertainty, fear of contracting the virus, frequent changes in workflow and staff deployment added stress to the participants.

In the multivariable model, predominantly work environment-related factors were associated with depression, anxiety and stress (Table 4). The only sociodemographic factors associated with psychological outcome in our study were female sex, which was associated with an increased risk of anxiety (adjusted OR 2.92, 95\% CI 1.49-5.72), and being married, which was associated with a decreased risk of anxiety (adjusted OR $0.63,95 \%$ CI $0.42-0.96$ ).

\section{DISCUSSION}

Our survey of frontline HCWs, performed midway through a nationwide lockdown in Singapore, revealed a high prevalence of depression (39.1\%), anxiety $(47.7 \%)$ and stress $(24.7 \%)$. Our study also highlights that despite the mild clinical impact on paediatric patients, the psychological impact on their healthcare providers was substantial. Factors associated with negative psychological impact were largely modifiable and included the requirement to perform additional tasks, lack of choice in work scope or environment, a perceived lack of protection from COVID-19, lack of access to physical activities, and experience of stigma from the community. Female sex was also associated with a higher risk of anxiety, whereas being married and working in the $\geq 71$-hour work week bracket seemed to be protective.

The baseline prevalence of major depressive disorder and generalised anxiety disorder in Singapore, based on the World Health Organization World Mental Health Composite International Diagnostic Interview criteria, was reportedly $5.8 \%$ and $0.9 \%$, respectively. ${ }^{25}$ In the early phase of the COVID-19 pandemic and prior to the nationwide lockdown, the prevalence of depression, anxiety and stress among $\mathrm{HCWs}$ was reported in a previous study to be $8.1 \%, 10.8 \%$ and $6.4 \%$, respectively. ${ }^{22}$ Our study indicates a sharp rise in prevalence of depression (39.1\%), anxiety (47.7\%) and stress $(24.7 \%)$ over the course of this pandemic. There were some differences between the prior study and ours; the former included subjects from adult-based hospitals and it is unclear if the participants worked in high-risk areas. It was also conducted approximately 2 months before our study when there were no reported COVID-19 deaths compared with 14 deaths by the time of our study. ${ }^{2}$ Hence, these results have to be interpreted in the context of the COVID-19 trajectory and the nationwide mitigation measures implemented in Singapore. ${ }^{18,19}$ During the lockdown period, a surge in the number of calls to the local national mental health hotline was reported, indicating that the prolonged mitigation measures could have an impact on mental health, and particularly depression. ${ }^{26} \mathrm{~A}$ Singapore report during the COVID-19 pandemic cited that the morale of HCWs was negatively affected by increased workload, uncertainty over the effectiveness of personal protective equipment, concerns of well-being of family members and stigmatisation from the public. ${ }^{15}$

Our study also highlights that paediatric HCWs are not exempt from the psychological stressors incurred during the COVID-19 pandemic. Despite the mild clinical impact on paediatric patients, the psychological impact on their healthcare providers was shown to be substantial. Firstly, being a frontline HCW itself may confer a higher risk of psychological impact compared with $\mathrm{HCWs}$ not working in high-risk areas. A multicentre study $(\mathrm{n}=1,257)$ conducted in China, which used the 9-item Patient Health Questionnaire, showed that frontline $\mathrm{HCWs}$ directly engaged in the diagnosis, treatment and care of patients with COVID-19 had 1.5 -fold increased odds of experiencing depression and anxiety. ${ }^{3}$ Secondly, the exposure to varying healthcare occupational hazards among paediatric HCWs (especially nursing staff) is known to be high, possibly associated with strained doctor-patientcaregiver relationships during stressful situations. ${ }^{27}$ A recent study examining the psychological impact on paediatric HCWs in China reported rates of depression, anxiety and stress of $15 \%, 18 \%$ and $10 \%$, respectively. ${ }^{10}$ It is concerning that our study reported rates that were greater than double the rates of the report: depression, 
Table 4. Risk factors for depression, anxiety and stress identified by multivariate analysis

\begin{tabular}{|c|c|c|}
\hline Characteristics & Adjusted odds ratio ( $95 \%$ confidence interval) & $P$ value \\
\hline \multicolumn{3}{|l|}{ Depression } \\
\hline \multicolumn{3}{|c|}{ Perceives a lack of choice in work scope or environment } \\
\hline Yes & $1.84(1.10-3.10)$ & 0.021 \\
\hline No & 1 [Reference] & \\
\hline \multicolumn{3}{|c|}{ Perceives a lack of access to physical activities } \\
\hline Yes & $2.81(1.78-4.42)$ & $<0.001$ \\
\hline No & 1 [Reference] & \\
\hline \multicolumn{3}{|c|}{ Experiences stigma from community } \\
\hline Yes & $2.51(1.54-4.08)$ & $<0.001$ \\
\hline No & 1 [Reference] & \\
\hline \multicolumn{3}{|l|}{ Anxiety } \\
\hline \multicolumn{3}{|l|}{ Sex } \\
\hline Female & $2.92(1.49-5.72)$ & 0.002 \\
\hline Male & 1 [Reference] & \\
\hline \multicolumn{3}{|l|}{ Married } \\
\hline Yes & $0.63(0.42-0.96)$ & 0.033 \\
\hline No & 1 [Reference] & \\
\hline Weekly work duration (hours) & & $0.035^{\mathrm{a}}$ \\
\hline$<40$ & 1 [Reference] & \\
\hline $40-50$ & $0.80(0.44-1.47)$ & 0.471 \\
\hline $51-60$ & $0.56(0.25-1.28)$ & 0.172 \\
\hline $61-70$ & $0.36(0.12-1.06)$ & 0.064 \\
\hline$\geq 71$ & $0.19(0.06-0.64)$ & 0.007 \\
\hline \multicolumn{3}{|c|}{ Perceives a lack of choice in work scope or environment } \\
\hline Yes & $2.01(1.16-3.50)$ & 0.013 \\
\hline No & 1 [Reference] & \\
\hline \multicolumn{3}{|l|}{ Perceives a lack of sufficient rest } \\
\hline Yes & $3.02(1.37-6.67)$ & 0.006 \\
\hline No & 1 [Reference] & \\
\hline \multicolumn{3}{|c|}{ Experiences stigma from community } \\
\hline Yes & $2.77(1.66-4.64)$ & $<0.001$ \\
\hline No & 1 [Reference] & \\
\hline \multicolumn{3}{|l|}{ Stress } \\
\hline \multicolumn{3}{|c|}{ Required to perform additional tasks } \\
\hline Yes & $1.84(1.04-3.27)$ & 0.036 \\
\hline No & 1 [Reference] & \\
\hline \multicolumn{3}{|c|}{ Perceives a lack of protection from COVID-19 } \\
\hline Yes & $2.22(1.15-4.29)$ & 0.018 \\
\hline No & 1 [Reference] & \\
\hline \multicolumn{3}{|c|}{ Perceives a lack of access to physical activities } \\
\hline Yes & $2.88(1.79-4.64)$ & $<0.001$ \\
\hline No & 1 [Reference] & \\
\hline
\end{tabular}

${ }^{a}$ Type $3 P$ value 
$39.1 \%$; anxiety, $47.7 \%$; and stress, $24.7 \%$. However, this discrepancy may be due to differences in study design; the earlier study only included medical professionals and utilised the social media platform that may have significantly biased the population surveyed. ${ }^{10}$

Symptoms of anxiety were reported in $25.5-44.6 \%$ of HCWs in China during the COVID-19 pandemic, which was comparable to results of our survey. ${ }^{3,4}$ Consistent with these studies, the female sex was associated with risk of anxiety. ${ }^{3}$ A concern is that the majority of medical and nursing staff in our centre were female (369 of 430, 85.8\%). Similarly, a Singapore study reported that $35.8 \%$ of antenatal women were screened positive for anxiety during the COVID-19 pandemic. ${ }^{28}$ Further research to identify actual anxiety disorders may be necessary as a next step to diagnose and support our staff. In our cohort, being married and working in the $\geq 71$-hour work week bracket seemed to be associated with a reduced risk of anxiety. The latter seems counterintuitive, but on examining the multivariable model, there was a consistent trend of decreased risk of anxiety with increased work hours per week. More investigation is needed to explore the association between working hours and psychological impact during a pandemic. A study performed during the severe acute respiratory syndrome (SARS) epidemic in Hong Kong alluded to a similar phenomenon; it reported that HCWs who were less willing to work in SARS units were at a higher risk for psychological morbidity, whereas those who volunteered to do so were more psychologically prepared and had better "reserves" to cope with the epidemic. ${ }^{29}$ Hence, we observed that participants who perceived a lack of choice in work scope or environment may be less psychologically resilient to challenges and were more vulnerable to psychological impact.

The prevalence of stress reported in our study was lower than that of other research involving $\mathrm{HCWs}$ or the general public during COVID-19 outbreak. ${ }^{3,6}$ Respondents reported that clear communication from hospital leaders, availability of adequate personal protective equipment, support from the hospital administration, colleagues, family and religious observances helped relieve stress during this pandemic. As initially described in the SARS study in Hong Kong, our report confirms that psychological support from employers has a protective effect against stress in the workplace. ${ }^{29}$ The perception of a lack of protective gear influenced the risk of stress in HCWs (adjusted relative risk $2.22,95 \%$ CI $1.15-4.29, P=0.0177$ ). The reassurance of personal safety and availability of adequate personal protective equipment during the COVID-19 pandemic are factors that encourage medical staff to continue working during the pandemic. ${ }^{4,30}$ It is known that COVID-19 is highly infectious; therefore, reassurance from clear infectious control guidelines is important to ensure the psychological well-being of HCWs.

This study adds to the existing but limited literature on the psychological impact of the COVID-19 pandemic on paediatric HCWs. Our study has several limitations. As with every self-reporting questionnaire, responses elicited may be biased in favour of finding a certain outcome rather than representing what the participants truly believe. ${ }^{31}$ Measures were taken to minimise this bias; for example, the questionnaires were administered in an anonymous manner and a high response rate minimised selection bias. Secondly, symptoms of depression, anxiety and stress identified in the DASS-21 are not equivalent to a formal psychiatric assessment and do not constitute a diagnosis according to the Diagnostic and Statistical Manual of Mental Disorders. ${ }^{32}$ Thirdly, this survey sampled participants from a single site and therefore has limited generalisability. Lastly, this cross-sectional study lacks baseline and longitudinal comparison. Nevertheless, our study provides preliminary data that future research is necessary to track progression or resolution of mental health symptoms of HCWs as the COVID-19 pandemic situation changes, and to evaluate the effects of any therapeutic intervention.

\section{CONCLUSION}

After 3 months into the COVID-19 pandemic and midway into a nationwide lockdown, approximately $40 \%$ of high-risk paediatric HCWs reported symptoms of depression and anxiety. It is necessary to track progression or resolution of mental health symptoms in this cohort as the COVID-19 pandemic situation evolves, and for hospitals to consider interventions to support the mental wellness of HCWs. Personal psychoneuroimmunity prevention measures, including hand hygiene and wearing of face masks, as well as organisational measures including stepping-up of workplace hygiene measures and support from the hospital management, can be implemented to lessen psychiatric symptoms. At the national level, involving mental health professionals to plan and coordinate psychological intervention for the country should be considered. This is especially pertinent, given that the pandemic is likely to be long drawn out and affecting many facets of healthcare. 


\section{REFERENCES}

1. World Health Organization. Timeline: WHO's COVID-19 response. Available at: https://covid19.who.int/. Accessed on 25 March 2021.

2. Worldometer. Coronavirus updates. Available at: https://www. worldometers.info/coronavirus/. Accessed on 25 March 2021.

3. Lai J, Ma S, Wang Y, et al. Factors associated with mental health outcomes among health care workers exposed to coronavirus disease 2019. JAMA Netw Open 2020;3:e203976.

4. Lu W, Wang H, Lin Y, et al. Psychological status of medical workforce during the COVID-19 pandemic: A cross-sectional study. Psychiatry Res 2020;288:112936.

5. Sim MR. The COVID-19 pandemic: Major risks to healthcare and other workers on the front line. Occup Environ Med 2020;77:281-2.

6. Wang $\mathrm{C}$, Pan $\mathrm{R}$, Wan X, et al. Immediate psychological responses and associated factors during the initial stage of the 2019 coronavirus disease (COVID-19) epidemic among the general population in China. Int J Environ Res Public Health 2020;17:1729.

7. Chew NWS, Ngiam JN, Tan BY, et al. Asian-Pacific perspective on the psychological well-being of healthcare workers during the evolution of the COVID-19 pandemic. BJPsych Open 2020;6:e116.

8. Chew NWS, Lee GKH, Tan BYQ, et al. A multinational, multicentre study on the psychological outcomes and associated physical symptoms amongst healthcare workers during COVID-19 outbreak. Brain Behav Immun 2020;88:559-65.

9. Dong Y, Mo X, Hu Y, et al. Epidemiology of COVID-19 among children in China. Pediatrics 2020;145:e20200702.

10. Liu Y, Wang L, Chen L, et al. Mental health status of paediatric medical workers in China during the COVID-19 outbreak. Front Psychiatry 2020;11:702.

11. Kam KQ, Yung CF, Cui L, et al. A well infant with coronavirus disease 2019 with high viral load. Clin Infect Dis 2020;71:847-9.

12. Ministry of Health, Singapore. Updates on COVID-19 (coronavirus disease 2019) local situation. Available at: https:/www.moh.gov.sg/ covid-19. Accessed on 25 March 2021.

13. Eysenbach G. Improving the quality of Web surveys: The Checklist for Reporting Results of Internet E-Surveys (CHERRIES). J Med Internet Res 2004;6:e34.

14. Ho CS, Chee CY, Ho RC. Mental health strategies to combat the psychological impact of COVID-19 beyond paranoia and panic. Ann Acad Med Singap 2020;49:155-60.

15. Liew MF, Siow WT, MacLaren G, et al. Preparing for COVID-19: Early experience from an intensive care unit in Singapore. Crit Care 2020;24:83

16. Phua J, Weng $\mathrm{L}$, Ling $\mathrm{L}$, et al. Intensive care management of coronavirus disease 2019 (COVID-19): Challenges and recommendations. Lancet Respir Med 2020;8:506-17.

17. Tan RMR, Ong GY, Chong SL, et al. Dynamic adaptation to COVID-19 in a Singapore paediatric emergency department. Emerg Med J 2020;37:252-4.
18. Tan THY, Toh MPHS, Vasoo S, et al. Coronavirus disease 2019 (COVID-19): The Singapore experience. A review of the first eight months. Ann Acad Med Singap 2020;49:764-78.

19. Chen JI, Yap JC, Hsu LY, et al. COVID-19 and Singapore: From early response to circuit breaker. Ann Acad Med Singap. 2020;49:561-72.

20. Lovibond SH, Lovibond PF. Manual for the Depression Anxiety Stress Scales. 2nd ed. Sydney: Psychology Foundation; 1995.

21. Antony MM, Bieling PJ, Cox BJ, et al. Psychometric properties of the 42-item and 21-item versions of the Depression Anxiety Stress Scales in clinical groups and a community sample. Psychol Assess 1998;10:176-81.

22. Tan BYQ, Chew NWS, Lee GKH, et al. Psychological impact of the COVID-19 pandemic on health care workers in Singapore. Ann Intern Med 2020;173:317-20.

23. Tee ML, Tee CA, Anlacan JP, et al. Psychological impact of COVID-19 pandemic in the Philippines. J Affect Disord 2020; 277:379-91.

24. Lovibond PF, Lovibond SH. The structure of negative emotional states: Comparison of the Depression Anxiety Stress Scales (DASS) with the Beck Depression and Anxiety Inventories. Behav Res Ther $1995 ; 33: 335-43$

25. Chong SA, Abdin E, Vaingankar JA, et al. A population-based survey of mental disorders in Singapore. Ann Acad Med Singap 2012;41:49-66.

26. The Straits Times. Mental health fallout: How Covid-19 has affected those in Singapore, 9 May 2020. Available at: https://www. straitstimes.com/singapore/health/mental-health-fallout. Accessed on 28 November 2020.

27. Ma Y, Ni X, Shi Y, et al. Epidemic characteristics and related risk factors of occupational exposure for pediatric health care workers in Chinese public hospitals: A cross-sectional study. BMC Public Health 2019;19:1453

28. Ng QJ, Koh KM, Tagore S, et al. Perception and feelings of antenatal women during COVID-19 pandemic: A cross-sectional survey. Ann Acad Med Singap 2020;49:543-52.

29. Tam CW, Pang EP, Lam LC, et al. Severe acute respiratory syndrome (SARS) in Hong Kong in 2003: Stress and psychological impact among frontline healthcare workers. Psychol Med 2004;34:1197-204.

30. Cai H, Tu B, Ma J, et al. psychological impact and coping strategies of frontline medical staff in Hunan between January and March 2020 during the outbreak of coronavirus disease 2019 (COVID19) in Hubei, China. Med Sci Monit 2020;26:e924171.

31. Althubaiti A. Information bias in health research: Definition, pitfalls, and adjustment methods. J Multidiscip Healthe 2016;9:211-7.

32. American Psychiatric Association. Diagnostic and Statistical Manual of Mental Disorders (DSM-5). 5th ed. Arlington, VA: American Psychiatric Association; 2013. 